\title{
Empirical Studies Concerning the Maintenance of BPMN Diagrams: A Systematic Mapping Study
}

\author{
Ursula Campos, Adriana Lopes and Tayana Conte \\ USES Research Group \\ Institute of Computing, Federal University of Amazonas \\ Manaus, AM - Brazil \\ \{usc, adriana, tayana\}@icomp.ufam.edu.br
}

\author{
Simone Diniz Junqueira Barbosa \\ Informatics Department \\ PUC-Rio \\ Rio de Janeiro, Brazil \\ simone@inf.puc-rio.br
}

\begin{abstract}
Business process models help understand the organizational process and the software that supports it. BPMN (Business Process Modeling and Notation) is the standard notation for business process modeling, and it is widely accepted in industry. BPMN models can elucidate the activities carried out by a software during its construction and maintenance. However, during the maintenance of the software that supports an organizational process, usually only the source code of the software undergoes modifications, even when inserting new features. The software design models, including the BPMN models, often become outdated over time and, in future maintenance, they will not help understand the business process in which the software is inserted and which the software aims to support. Such scenario highlights the importance of supporting the maintenance of BPMN models. However, what has been experimentally investigated regarding the maintenance of BPMN models? To answer this question, we performed a systematic mapping, which showed experimental studies, factors and technologies that influence the maintenance of BPMN models. These results present conclusions about the state of the art and gaps that can be explored in this field of research.
\end{abstract}

Keywords - Business Process Model and Notation; BPMN; Software Maintenance; Model Maintenance; Systematic mapping.

\section{INTRODUCTION}

Business process modeling is an essential activity for the success of business process management (BPM) [1]. The BPMN (Business Process Modeling and Notation) is the standard notation maintained by OMG [2] for business process modeling and is widely accepted in industry [3]. Researchers and practitioners recognize that understanding the business processes is key to identifying the users' needs that a software should support [4]. Martinez et al. [5] emphasize that the focus on software development from business processes can increase the software's compliance with the needs of its users. BPMN models can help in understanding the activities carried out by the software and better support software maintenance.

Maintenance is one of the most crucial phases of the software life cycle. It is usually divided into two steps: understanding the software artifact and modifying the software artifact [6]. In fact, a software artifact should be well understood before being modified, because developers need to know what impact modifications will have on the software and, possibly, on the underlying business processes. In the maintenance stage, process models are of great relevance in understanding the software being developed [7] or modified. Thus, it is important that they are consistent with the current version of the software, so that it is understood correctly.

However, during the software maintenance process, usually only the source code is modified, even when inserting new features. Over time, development team turnovers mean that new members may not know the software functionalities, since they did not participate in its design and development [8]. It is in this scenario that software models prove to be of great value, because developers first seek to understand the software functionalities through the models built during its project. However, the documentation is almost always outdated and inconsistent [9]. When the models are not maintained together with the software, the information in these models will be inconsistent with the current version of the software, which hinders new professionals' understanding and activities during software maintenance.

As we realize the importance of supporting the maintenance of BPMN models, it is important to know what has been experimentally investigated on the maintenance of BPMN models. The goal of this paper is to describe a systematic mapping of studies related to the maintenance of BPMN models in order to identify what has been done in the literature to support and facilitate the maintenance of such models. The systematic mapping revealed factors and technologies related to the context of maintenance of BPMN models. With this work we present conclusions about the state of the art and gaps that can be explored in this field of research.

The remainder of this paper is organized as follows: Section 2 presents the related works. Section 3 describes the methodology applied to conduct the systematic mapping. Section 4 presents the results of the mapping study. Section 5 discusses the threats to the validity of this work. Finally, Section 6 presents the conclusions of the systematic mapping.

\section{RELATED WORKS}

In this section, we present some related works to our work. Pourmirza et al. [10] present a systematic literature review of Business Process Management Systems (BPMS) architectures. BPMS are information systems that interpret business processes to ensure that the activities specified therein are properly 
executed and monitored by an organization. In this work, BPMS architectures that served as primary studies were compared with respect to the reference architecture that they are based on, the level of detail at which they are described, the architectural styles that they use, the means with which they are evaluated, and the functionality that they support. The resulting comparison provides an overview of and insights into the current body of knowledge on BPMS architectures.

Valença et al. [11] present a systematic mapping study of business process variability approaches, which is an emergent field in BPM with many of its proposals inspired by theories from Software Product Line to handle process variability. According to the authors, variability in business processes is necessary for organizations dealing with environmental changes. The results show that a significant number of approaches is available, but most of them lack empirical studies.

Although these works deal with BPM, they do not exploit the research done on the maintenance of BPMN models. Our work investigates the maintenance of BPMN models in the literature.

\section{RESEARCH METHOD}

A systematic mapping is a broad review of primary studies in a specific topic area, aiming to identify what evidence is available on the topic [12]. We followed the guidelines proposed by Kitchenham and Charters [12]. The following subsections detail our systematic mapping protocol.

\section{A. Goal}

We had the following goals for the systematic mapping:

- To investigate the maintenance of BPMN models and whether the models are understandable and modifiable to allow them to be modified while maintaining the source code.

- To gather experimental evidence on the use of BPMN models in their maintenance or use during the maintenance of the software source code.

\section{B. Research Question}

This mapping aimed to answer the following research question: "What has been experimentally investigated regarding the maintenance of BPMN models?". In order to answer this question, we divided the systematic mapping into specific sub-questions about the maintenance of BPMN models (see Table I).

TABLE I. RESEARCH SUBQUESTS

\begin{tabular}{|c|l|}
\hline Subquestion & \multicolumn{1}{|c|}{ Description of Ssubquestion } \\
\hline SQ1 & $\begin{array}{l}\text { What is the state of the art in experimental studies on } \\
\text { maintenance of BPMN models or source code } \\
\text { maintenance when using BPMN models? }\end{array}$ \\
\hline SQ2 & $\begin{array}{l}\text { Which dependent variables are investigated in the } \\
\text { experimental studies? }\end{array}$ \\
\hline SQ3 $^{1}$ & $\begin{array}{l}\text { Which of the factors studied influence the software } \\
\text { maintenance capability (source code or model)? }\end{array}$ \\
\hline SQ4 & $\begin{array}{l}\text { What technologies support the maintenance of BPMN } \\
\text { models? }\end{array}$ \\
\hline
\end{tabular}

\section{Search strategy}

To construct the search string, we defined the search terms based on the procedure described by Kitchenham and Charters [12], who suggested defining the parameters for Population, Intervention, Comparison, Result, and Context (PICOC). The population was the specific field of research on BPMN Business Process Modeling and Notation; the intervention was composed of maintenance phases or types; the result was the types of experimental studies; comparison and context were not applicable because our goal is to characterize what is done in relation to the maintenance of BPMN models, so there is no comparison to determine the context. We have identified the terms of the research through the publication of Fernandez-Saez et al. [6], which we used as the basis for this work. They investigated the maintenance of UML models, so we adapted this work by bringing it into the context of BPMN models. Table II shows the terms used in the search string and the groups of synonyms used in its construction. We used the boolean operator OR between the alternative terms and synonyms, and the Boolean operator AND to join the groups.

We used the search string in the Scopus ${ }^{2}$, Engineering Village $^{3}$ and $\mathrm{ACM}^{4}$ digital libraries. We have included the Scopus and Engineering Village libraries because they are meta libraries and index publications from several reputable publishers in Software Engineering, such as ACM, IEEE, Springer and Elsevier, and they allow defining filters by type of document, language and area of knowledge. Although the ACM library is indexed by Scopus, we included this library to ensure that there were no excluded publications in the Scopus indexing and because ACM indexes some Springer Link and Science Direct publications as well.

TABLE II. SEARCH STRING TERMS AND SYNONYMS

\begin{tabular}{|c|c|l|}
\hline $\begin{array}{c}\text { Term of } \\
\text { PICOC }\end{array}$ & Main Term & \multicolumn{1}{c|}{ Synonyms } \\
\hline Population & BPMN & $\begin{array}{l}\text { business process model and notation } \\
\text { OR } \\
\text { business process modeling and } \\
\text { notation }\end{array}$ \\
\hline Intervention & Maintenance & $\begin{array}{l}\text { evolution OR comprehension OR } \\
\text { maintainability OR evolvability OR } \\
\text { understandability OR modularity OR } \\
\text { modification OR understanding OR } \\
\text { reusability OR stability OR } \\
\text { misinterpretation OR analyzability OR } \\
\text { testability OR changeability OR } \\
\text { comprehensibility }\end{array}$ \\
\hline Results & Empirical & $\begin{array}{l}\text { survey OR action research OR } \\
\text { experiment OR case study }\end{array}$ \\
\hline
\end{tabular}

\section{Selection Criteria}

The selection process comprised three steps (first filter, second filter and process of extracting data). In the first filter, two researchers only read the title and the abstract. They have selected the publications applying the inclusion and exclusion criteria (see Table III). The reliability of the inclusion and

\footnotetext{
1 We considered as factors the results of the publications selected in the

${ }^{3}$ http://www.engineeringvillage.com

systematic mapping that influence the maintenance of BPMN models.

${ }^{2}$ http://www.scopus.com

4 http://dl.acm.org
} 
exclusion criteria of a publication in the systematic mapping was assessed by applying Fleiss' Kappa [13]. Fleiss' Kappa is a statistical measure for assessing the reliability of agreement between a fixed number of raters when classifying items.

TABLE III. INCLUSION AND EXCLUSION CRITERIA

\begin{tabular}{|c|c|}
\hline$\#$ & Selection Criteria \\
\hline IC1 & $\begin{array}{l}\text { Papers with experimental studies using BPMN model during } \\
\text { the maintenance of the diagram or source code }\end{array}$ \\
\hline IC2 & $\begin{array}{l}\text { Papers with experimental studies with BPMN models } \\
\text { helping in the process of understanding the software }\end{array}$ \\
\hline IC3 & $\begin{array}{l}\text { Papers that evaluate the comprehensibility or maintainability } \\
\text { of BPMN diagrams }\end{array}$ \\
\hline \# & Exclusion Criteria \\
\hline $\mathrm{EC} 1$ & Papers proposing BPMN extensions; \\
\hline $\mathrm{EC} 2$ & Papers that do not report experimental studies; \\
\hline EC3 & $\begin{array}{l}\text { Papers that mention BPMN or maintenance only as general } \\
\text { introductory terms in the abstract and nowhere else; }\end{array}$ \\
\hline $\mathrm{EC} 4$ & Papers that are not written in English or Portuguese; \\
\hline EC5 & $\begin{array}{l}\text { Papers unavailable for reading or data collection (paid } \\
\text { publications, broken links in the search engine and not made } \\
\text { available by the authors after an attempt to contact); }\end{array}$ \\
\hline EC6 & Duplicate papers \\
\hline
\end{tabular}

Initially, we asked two researchers to classify, individually, a random sample of 20 publications to analyze the degree of agreement in the selection process through the Fleiss' Kappa [13]. The selected sample was the set of the publications returned by Scopus. The result of the degree of agreement showed a substantial level of agreement between the two researchers $($ Kappa $=0.653)$.

In the second filter, the researchers fully read the selected publications, selecting them according to the same criteria used in the first filter. After completing the selection process, we started the process of extracting data.

We used an extraction form to standardize the data collected. According to Fernandez et al. [6], this ensures that the same criteria will be used, thus facilitating their classification. We extracted the data according to each subquestion. The complete protocol and the publications obtained in the second filter are available in a technical report [14].

\section{RESULTS}

Figure 1. depicts the publications selection process carried out in the conduction of the systematic mapping. The search string returned 89 publications in Scopus library, 80 in Engineering Village, and 19 in ACM. After eliminating duplicates, we had 88 publications in Scopus, 7 in Engineering Village and 15 in ACM, resulting in 110 publications. During the first filter, we rejected 81 publications that did not meet the inclusion criteria. We read the remaining 29 publications in full and classified them in the second filter. At that stage we selected 18 publications that proceeded to the extraction process.

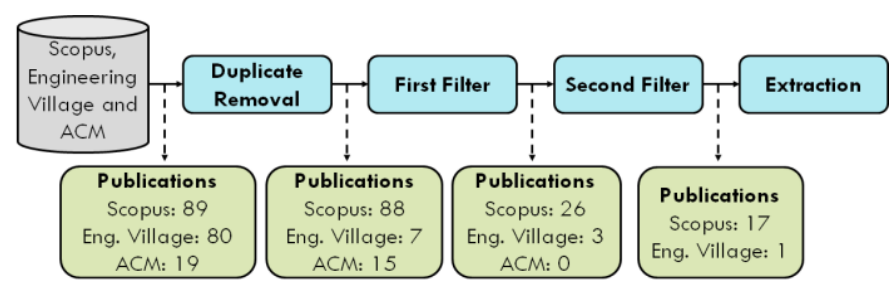

Figure 1. Article selection process.

The selected papers were published between 2008 and 2018 . There was no criterion to limit the year of publications. Figure 2. shows that most publications are recent, which leads us to believe that the maintenance of BPMN models is a timely research issue.

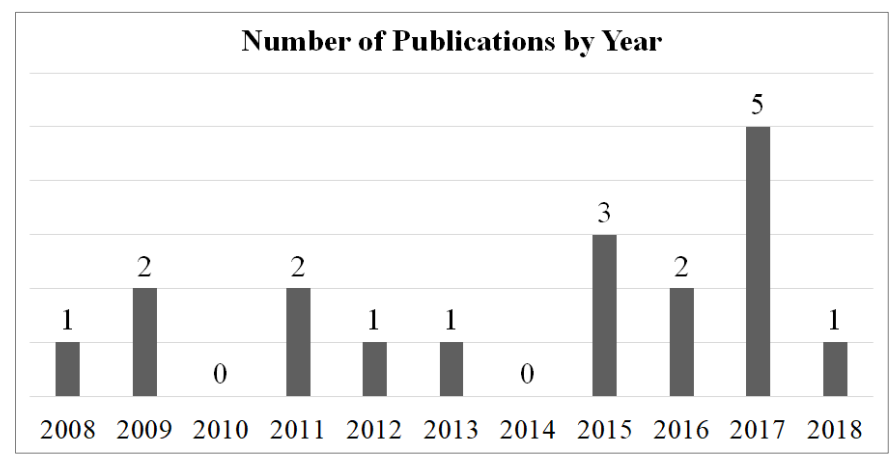

Figure 2. Overview of the publications per year.

The answers we found for each research sub-question are the following:

1) SQ1: What is the state of the art in experimental studies on maintenance of BPMN models or source code maintenance when using BPMN models?

This subsection presents several items related to the state of the art of experimental studies regarding the maintenance of BPMN models. We present these items next:

Experimental Study Type: Figure 3. shows the number of publications with each type of experimental study. Even when a publication reported more than one controlled experiment, i.e., replications of the experiment in other institutions or with other artifacts, Figure 3. only counts it once.

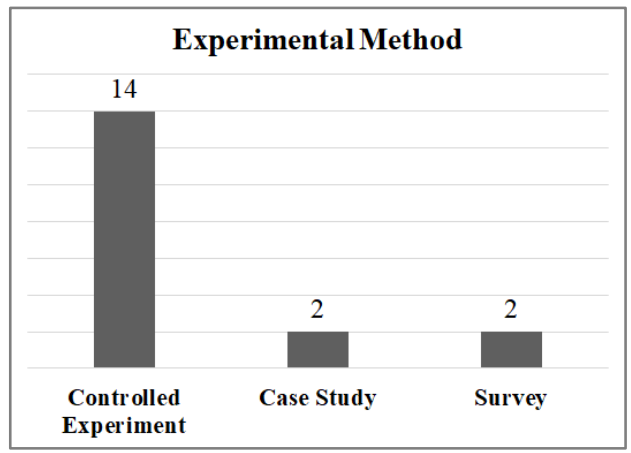

Figure 3. Types of experimental studies reported in the publications.

Experimental studies: When counting the number of experimental studies reported in the 18 publications, according to each type of experiment. If one publication reported two 
controlled experiments, we counted the two experiments. In total, the 18 publications presented 39 experimental studies: 35 controlled experiments, 2 case studies, and 2 surveys.

Context: We classified the context of the studies as laboratory, industry or online. Most publications (72.22\%) reported experiments that were conducted in a laboratory within academic environment. Two publications (11.11\%) reported experiments carried out in industry (in companies that use BPMN models). Three publications (16.67\%) reported experiments performed online, where a task was made available online and the participant had a deadline to do it (e.g., a week). We can see that the amount of experiments performed in industry is low, which corroborates the result already obtained in the previous section, about the need to perform experiments in real environments.

Characterization of the participants: Most publications have presented experimental studies conducted with undergraduate or graduate students, which is not necessarily inappropriate, since student skills are considered similar to the skills of novice practitioners [15][16]. Some publications have conducted experiments with more than one participant profile. Among the 39 experiments presented in the publications, 29 were experiments with only students as subjects, 5 were experiments with only professionals, 3 were experiments with students and professionals, 1 experiment with students, professionals and academic professionals, and 1 experiment in which the participant was the author of the technique. In the latter, the author applied the technique proposed by himself, to reduce the complexity of BPMN models.

Maintenance focus object: Software maintenance tasks usually require some modification in the source code, and should be accompanied by modifications in the corresponding BPMN model. We did not find experimental studies that reported the use of BPMN models during source code maintenance. We only found publications where the maintenance was done only in the BPMN model, consisting mostly of experimental studies that evaluate the comprehensibility of BPMN models.

Treatments in experimental studies: Figure 4. shows the different treatments used in the experimental studies. We divided the treatments into six categories: (i) complexity of the model, (ii) model representation, (iii) model characteristics, (iv) type of model representation, (v) model representation method, and (vi) characteristics of the model maintainers and defects in the models.

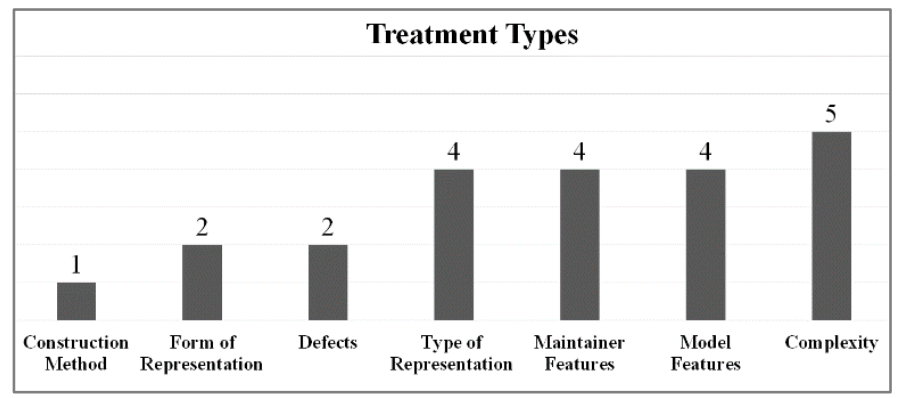

Figure 4. Types of treatment in experiments, by publication.
We highlight the category complexity of the model with the highest number of publications. Some publications presented experiments with more than one type of treatment, thus the bars add up to 22 publications. The types of treatment of each publication are more detailed in a technical report [14].

According to the data obtained, we can conclude the following about the state of the art of research on the maintenance of BPMN models:

Most of the experimental studies carried out were controlled experiments, carried out in the laboratory, with the participation of students.

Various types of treatment were applied such as: the complexity of the models, the form of representation, the characteristics of the models, among others.

The focus of the maintenance was always the model itself not coupled with source code maintenance.

2) SQ2: Which dependent variables are investigated in the experimental studies?

The dependent variables we investigated in the experimental studies are represented in Figure 5. We identified four dependent variables: model understanding, model modifiability, model complexity, and model completeness (i.e., to what extent the model represented the functionalities of a specific process). We can see that the great majority of publications sought to evaluate the comprehension of BPMN models (17), some publications evaluated the modifiability (4) and complexity (4) of the models and only one publication evaluated the completeness of the BPMN models. Some papers investigated both the comprehension and the modifiability or complexity of BMPN models in the same experiment, so the bars add up to 26 .

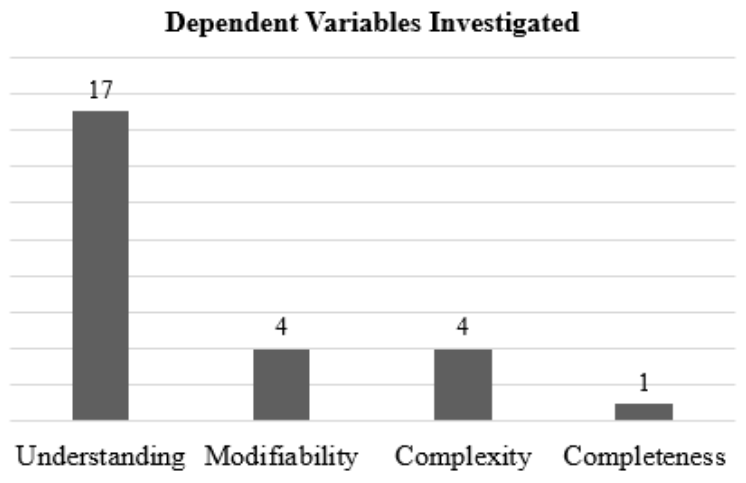

Figure 5. Dependent variables investigated.

3) SQ3: Which of the factors studied influence the software maintenance capability (source code or model)?

As we have already mentioned, we have not found experimental studies that dealt with the maintenance of the source code and BPMN together. This research subquestion will therefore focus only on the model. Figure 6 . shows the factors that influence, either positively or negatively, the maintenance of BPMN models, according to the results obtained in the experiments of the selected publications (publications are called PB\#, such as PB1 for the first publication in the report [14]). 


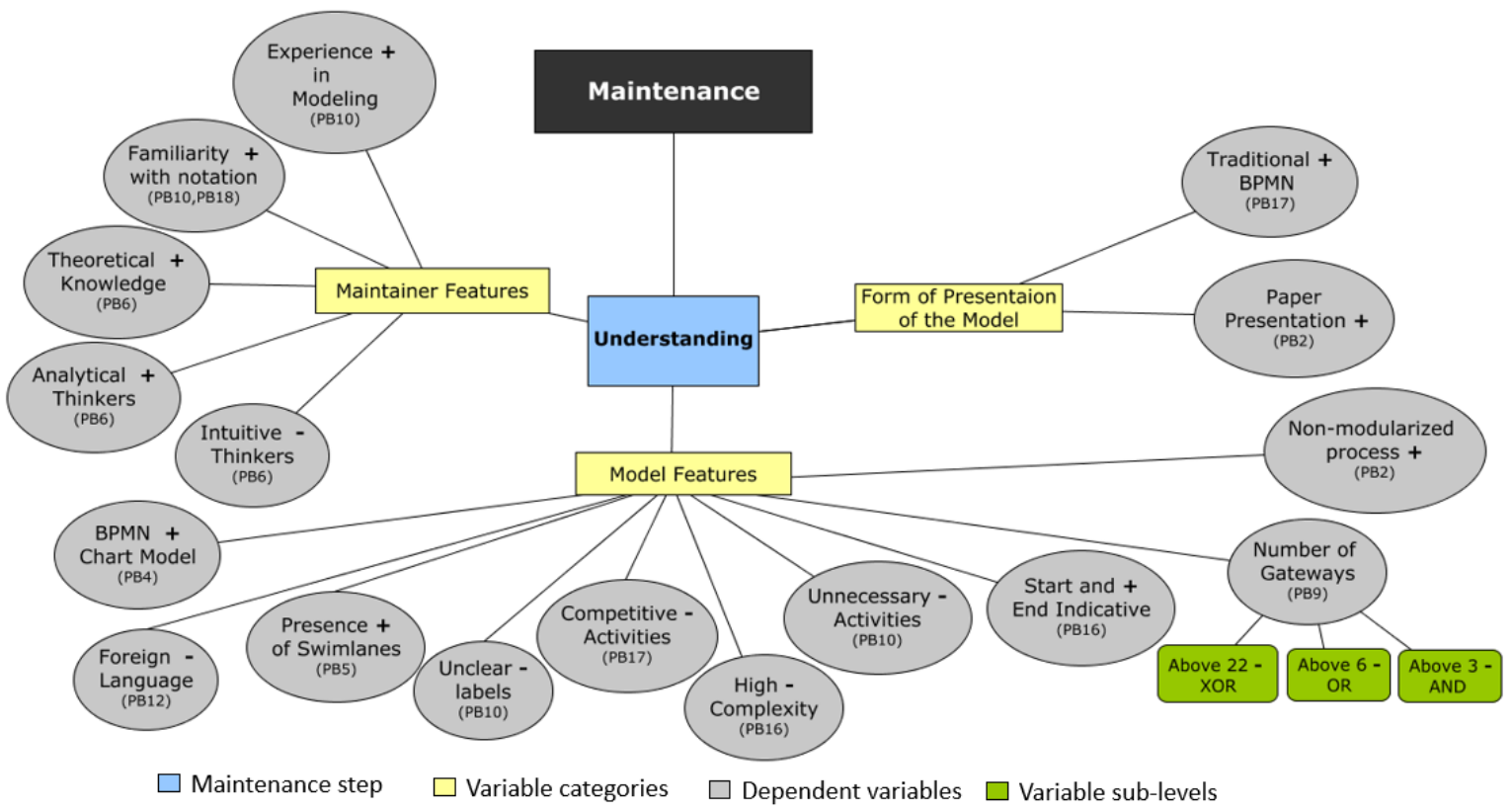

Figure 6. Dependent variables per publication.

It is important to remember that the maintenance activity is divided into two tasks [6]: artifact understanding and artifact modification. Considering these two types of maintenance tasks, the factors identified in the results of the experiments were related to understanding (see Figure 6. ). The experiments that dealt with modifying the models actually had some kind of technology to support the modification and were therefore fitted as a response to SQ4. Factors that did not present significant results (if the study could not determine whether a factor influenced or not the understanding of a BPMN model) were omitted. We classified as positive (+) the factors that facilitate the understanding of the model and as negative (-) the factors that undermine the understanding of the model.

\section{4) SQ4: What technologies support the maintenance of BPMN models?}

We divided the technologies that support the maintenance of models according to the two types of maintenance tasks: understanding and modification. We then evaluated whether the proposed technology supported understanding or modifying the model during maintenance. Figure 7. shows the technologies identified to support the maintenance of BPMN models.

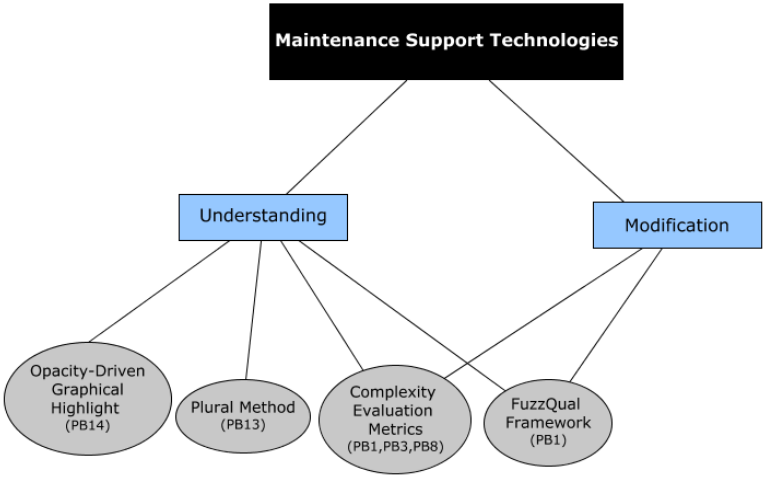

Figure 7. Technologies that support the maintenance of BPMN models
Technologies aimed at supporting the maintenance of BPMN models that focused on understanding were as follows:

- Opacity-Driven Graphical Highlights Technique: Jost and Hericko's approach [17] makes business process models less complex without changing the notation. The technique consists of working on the opacity of the models, highlighting only the relevant parts of the model according to the context in which it is used.

- Plural Method: decentralized method for creating BPMN models, in which the sources of model construction are also participants in the process. The method aims to involve process participants in modeling, since they are well aware of the problem domain.

Technologies to support the maintenance of BPMN models that focused on understanding and modification were as follows:

- Complexity measurement metrics: measures that may be useful in forecasting different aspects of understanding and modifying business process models in future model maintenance. The goal is to make models easier to understand and modify for all stakeholders.

- FuzzQual Framework: approach that evaluates BPMN models with regard to comprehensibility and modifiability. A framework/system built in JAVA evaluates the model based on the complexity metrics chosen, among several metrics that were evaluated. Through fuzzy logic, the framework evaluates the model with classifications of the type: "Moderately difficult to understand with a degree of certainty of $63 \%$ ", "Moderately difficult to modify with a degree of certainty of $100 \% "$. This framework is an implementation of the complexity metrics to evaluate BPMN models.

\section{THREATS TO VALIDITY}

Although we conducted this research under a systematic mapping methodology by defining a research protocol, some 
threats to validity can be identified: (i) the researchers' bias regarding the analysis of the primary studies; (ii) the university's limited access to some scientific databases, which can prevent some publications from being accessed; (iii) the limitation of the scope of this research to the selected databases. These threats were minimized by taking some actions. For the first threat, we reviewed the review protocol and performed the Kohen's Kappa statistical test in order to reduce the researchers' bias. Additionally, another experienced researcher reviewed the execution process. For the second threat, we had two publications that fit that threat. We requested the authors for the full publication whenever possible and included those that have been made available. Regarding the third threat, although the research was conducted in only three databases, they index publications from a large number of well-known publishers, journals and conferences.

\section{CONCLUSIONS}

This work describes the results of the systematic mapping of the literature that we carried out to identify what has been experimentally investigated on the maintenance of BPMN models. The main conclusions we have reached with the systematic mapping can be summarized as follows:

- Most studies were carried out in academic environments, which is explained by the fact that they are the most accessible environment for researchers. The difficulty to perform research in real environments, within industry/companies, is well known. However, there is a need for further experiments in real environments.

- Most studies performed are controlled experiments. This demonstrates a need for more case studies to confirm the results obtained in real environments.

- We have not identified studies that explored the maintenance of BPMN models together with maintenance of the software itself. We have also not identified studies that investigate the impact of updated or outdated BPMN models during software maintenance.

- The focus of the experimental studies is almost entirely on the understanding of BPMN models. However, understanding is only one of the tasks of maintenance, and it is necessary to focus also on modifying the models themselves.

- The technologies that we have found focus mostly on the initial construction of the model and how it will be easily understood or modified when it is necessary to use it or modify it. The proposed technologies seek to measure in advance whether the model created will be easy to maintain in the future. However, it is also necessary to create technologies that directly support the maintenance phase of the model.

The results indicate that there is a lack of maintenance of BPMN models, especially the maintenance of these models in conjunction with maintenance of the software source code. In relation to the technologies proposed, most of the research identified focuses on understanding the models, which is the initial task of maintenance, highlighting the need for technologies that support the modification of BPMN models.
This shows research opportunities to be explored in this field of research in future work.

\section{ACKNOWLEDGMENTS}

We thank all the students who participated in this research. We also thank the financial support granted by CAPES process 175956/2013, FAPEAM through Edital 009/2017; CNPq processes 311494/2017-0, 430642/2016-4, 423149/2016-4, and 311316/2018-2.

\section{REFERENCES}

[1] O. Turetken, T. Tompen, I. Vanderfeesten, A. Dikici and J. Van Moll, "The effect of modularity representation and presentation medium on the understandability of business process models in BPMN", Lecture Notes in Computer Science, vol. 9850, Springer, Cham, 2016.

[2] OMG, "Business process model and notation (BPMN)", v. 2.0, 2011.

[3] Z. Bukhsh, Z. Sinderen, N. Sikkel, and D. Quartel, "Understanding Modeling Requirements of Unstructured Business Processes", 2017, pp. 17-27.

[4] Shishkov, B., Xie, Z., Liu, K., and Dietz, J. L. (2002). "Using norm analysis to derive use cases from business processes". In Proceedings of the 5th Workshop On Organizational Semiotics.

[5] A. Martinez, J. Castro, O. Pastor, H. Estrada, "Closing the gap between organizational modeling and information system modeling". Workshop de Engenharia de Requisitos, Piracicaba, São Paulo-SP, 2003, pp. 93-108.

[6] A. Fernández-Sáez, M. Genero, M. R. V. Chaudron, "Empirical studies concerning the maintenance of UML diagrams and their use in the maintenance of code: A systematic mapping study", Information and Software Technology 55, 2013, pp. 1119-1142.

[7] E. Cruz, R. Machado, M. Santos, "Bridging the Gap between a set of interrelated Business Process Models and Software Models", 17th International Conference on Enterprise Information Systems (ICEIS), 2015, pp.338- 345 .

[8] E. Arisholm, L.C. Briand, S.E.Hove, Y. Labiche, "The Impact of UML Documentation on Software Maintenance: An Experimental Evaluation", IEEE Transactions on Software Engineering v. 32, 2006, pp.365-381.

[9] A. Forward, T. Lethbridge, "The relevance of software documentation, tools and technologies: a Survey", In Proceedings of the 2002 ACM symposium on Document engineering, 2002, pp. 26-33.

[10] S. Pourmirza, S. Peters, R. Dijkman, and P. Grefen. A systematic literature review on the architecture of business process management systems. Information Systems, 66, 2017, pp.43-58.

[11] G. Valença, Alves, C. Alves, V. and N. Niu, "A systematic mapping study on business process variability", International Journal of Computer Science \& Information Technology, 5(1), 2013, p.1

[12] B. Kitchenham and S. Chartes, "Guidelines for performing systematic literature reviews in software engineering", EBSE Technical Report EBSE 2007, Durham.

[13] J.L. Fleiss, "Statistical Methods for Rates and Proportions", Second ed., John Wiley \& Sons, New York, 1981..

[14] U. Campos, A. Lopes, S. de Souza, T. Conte, , "A systematic mapping on Empirical Studies Concerning the Maintenance of BPMN Diagrams", TR-USES-2019-0002, 2019. Available online at: http://uses.icomp.ufam.edu.br/wp-content/uploads/2019/03/TR-USES2019-002.pdf

[15] D. Budgen, A. Burn, P. Brereton, B. Kitchenham, R. Pretorius, "Empirical evidence about the UML: a systematic literature review", Software: Practice and Experience, 2010.

[16] P. Brereton, B. Kitchenham, D. Budgen, M. Turner, M. Khalil, "Lessons from applying the systematic literature review process within the software engineering domain", Journal of Systems and Software 80, 2007, pp. 571583.

[17] G. Jošt, M. Heričko and G. Polančič, Softw Syst Model, 2017. https://doi.org/10.1007/s10270-017-0618-5 